$$
\begin{array}{r}
\text { Pontifícia Universidade Católica } \\
\text { do Rio de Janeiro }
\end{array}
$$

Carlos Aparecido Ferreira

\title{
Novo Modelo de Transformador com Tap Variável em Regime Permanente
}

\section{Tese de Doutorado}

Tese apresentada ao Programa de Pós-graduação em Engenharia Elétrica do Departamento de Engenharia Elétrica da PUC-Rio como parte dos requisitos parciais para obtenção do título de Doutor em Engenharia Elétrica.

Orientador: Prof. Ricardo Bernardo Prada 


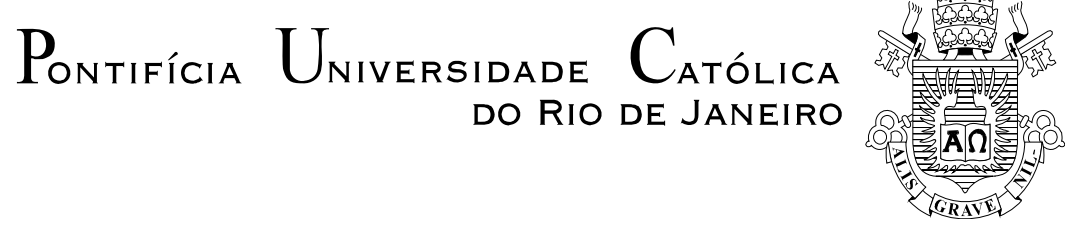

Carlos Aparecido Ferreira

Novo Modelo de Transformador com Tap Variável em Regime Permanente

Tese apresentada como requisito parcial para obtenção do grau de Doutor pelo Programa de Pós-Graduação em Engenharia Elétrica do Departamento de Engenharia Elétrica do Centro Técnico Científico da PUC-Rio. Aprovada pela Comissão Examinadora abaixo assinada.

Prof. Ricardo Bernardo Prada

Orientador

Departamento de Engenharia Elétrica - PUC-Rio

Prof. Vander Menengoy da Costa

UFJF

Prof. Luiz Claudio de Araujo Ferreira

Operador Nacional do Sistema Elétrico

Prof. Ricardo Mota Henriques Centro de Pesquisas de Energia Elétrica

Prof. Jorge Coelho

UFSC

Prof. Jorge Luiz de Araujo Jardim

UFF

Prof. Luiz Carlos Pereira da Silva

Unicamp

Prof. José Eugenio Leal

Coordenador Setorial do Centro

Técnico Científico

Rio de Janeiro, 30 de junho de 2011 
Todos os direitos reservados. É proibida a reprodução total ou parcial do trabalho sem autorização da universidade, do autor e do orientador.

\section{Carlos Aparecido Ferreira}

Graduado em Engenharia Elétrica pela Universidade Federal de Juiz de Fora (UFJF) em 2002, e Mestre em Engenharia Elétrica, também pela UFJF, em 2003. Atualmente é engenheiro dos quadros da Eletrobras.

Ficha Catalográfica

Ferreira, Carlos Aparecido

Novo modelo de transformador com tap variável em regime permanente / Carlos Aparecido Ferreira; orientador: Ricardo Bernardo Prada. - 2011.

195 f. ; $30 \mathrm{~cm}$

Tese (doutorado) - Pontifícia Universidade Católica do Rio de Janeiro, Departamento de Engenharia Elétrica, 2011.

Inclui bibliografia

1. Engenharia elétrica - Teses. 2. Transformador com tap variável. 3. LTC. 4. OLTC. 5. ULTC. 6. Estabilidade de tensão. 7. Modelo em regime permanente. I. Prada, Ricardo Bernardo. II. Pontifícia Universidade Católica do Rio de Janeiro. Departamento de Engenharia Elétrica. III. Título. 
Mesmo que eu tivesse o dom da profecia, e conhecesse todos os mistérios e toda ciência; mesmo que eu tivesse toda fé, a ponto de transportar montanhas, se não tiver amor não sou nada.

(Paulo de Tarso) 
Dedico esta tese aos meus pais: Carlos Humberto Ferreira e Neuza Maria de Lima Ferreira. 


\section{Agradecimentos}

Ao Deus salvador.

À intercessão de Nossa Senhora Aparecida.

Aos meus pais: Carlos Humberto Ferreira e Neuza Maria de Lima Ferreira pelo amor, sacrifícios e fé.

Aos meus irmãos: Carla, Cláudia e Caio e ao meu cunhado Luiz Carlos.

Ao meu orientador Ricardo Prada por todos os ensinamentos repassados ao longo desses anos, pela sabedoria acadêmica, segurança técnica, seriedade e atenção.

Às amizades que estão resistindo ao tempo, às distâncias, às diferenças, às dificuldades e às minhas indisponibilidades. Especialmente agradeço ao Pe. Paulo Alves Romão, pela disponibilidade em me ajudar no momento mais difícil.

Aos meus colegas da Eletrobras pelo apoio e incentivo durante a realização deste trabalho, especialmente aos amigos do Procel Indústria.

Ao Departamento de Planejamento de Transmissão da Eletrobras e à Eletrobras Cepel, pela utilização do software ANAREDE.

Aos meus demais familiares, especialmente à minha avó e madrinha Alaíde Ferreira Ruffo (in memorian) e aos meus primos Flavio de Melo Ferreira (in memorian) e Luiz Antonio Ferreira de Lima. 


\section{Resumo}

Ferreira, Carlos Aparecido; Prada, Ricardo Bernardo (Orientador). Novo Modelo de Transformador com Tap Variável em Regime Permanente. Rio de Janeiro, 2011. 195p. Tese de Doutorado - Departamento de Engenharia Elétrica, Pontifícia Universidade Católica do Rio de Janeiro.

O fenômeno de estabilidade de tensão vem despertando grande interesse acadêmico e das principais empresas de energia elétrica do mundo desde que começou a ser observado em sistemas reais, no final da década de setenta. Sua ocorrência está relacionada ao carregamento excessivo das linhas de transmissão. Modelar transformador com tap variável adequadamente é fundamental em análises de estabilidade de tensão, tanto no que diz respeito às informações fornecidas ao operador referentes às margens de estabilidade de tensão, quanto aos efeitos de ações de controle de tensão. O modelo de transformador com tap variável utilizado mundialmente consiste de uma impedância, obtida através do ensaio em curto-circuito e com tap nominal, em série com um transformador ideal. Esta tese mostra que, em estudos de estabilidade de tensão, o uso desse modelo leva a resultados qualitativamente errados. Para demonstração, utiliza-se um circuito pequeno e os conceitos de máxima potência transmitida, impedância equivalente da carga, e efeito do controle de tensão. Propõe-se um novo modelo coerente com os resultados obtidos em laboratório, com as leis de circuitos elétricos e com a teoria de estabilidade de tensão. Esse modelo pode ser utilizado em qualquer estudo em regime permanente. Através de diversas simulações computacionais, diferenças quantitativas e principalmente qualitativas foram obtidas comparando-se os resultados dos dois modelos.

\section{Palavras-chave}

Transformador com tap variável; LTC; OLTC; ULTC; estabilidade de tensão; modelo em regime permanente. 


\section{Abstract}

Ferreira, Carlos Aparecido; Prada, Ricardo Bernardo (Advisor). An Improved Steady-State Model for Tap-Changing Transformer. Rio de Janeiro, 2011. 195p. DSc. Thesis - Departamento de Engenharia Elétrica, Pontifícia Universidade Católica do Rio de Janeiro.

The voltage stability phenomenon is of interest since it began to be observed in real systems in the late seventies. It happens due to excessive loading of transmission lines. The modeling of tap-changing transformers is fundamental in voltage stability analysis, in terms of the information provided to the operator about voltage stability margins and the effects of voltage control actions. The model for tap-changing transformers currently in widespread use consists of an impedance, measured in a short-circuit test with a nominal tap, in series with an ideal transformer. The use of this model in voltage stability studies leads to qualitatively incorrect results, as shown in this thesis. For demonstration purpose a small circuit and the concepts of maximum load, equivalent load impedance and voltage control effects are used. An improved model that takes into account laboratory results, circuit laws and voltage stability theory is proposed. This model can be used in any steady-state study. It gives results that are not only more accurate than those obtained with the conventional model, but also, as shown in this thesis, qualitatively different.

\section{Keywords}

Tap-changing transformer; LTC; OLTC; ULTC; voltage stability; steadystate model. 


\section{Sumário}

1. Introdução $\quad 21$

1.1. Considerações Gerais 21

1.2. Motivação 22

1.3. Objetivo 22

1.4. Estrutura da Tese 23

2. Estabilidade de Tensão $\quad 25$

2.1. Caracterização do Fenômeno de Estabilidade de Tensão 25

$\begin{array}{ll}2.1 .1 \text {. Introdução } & 25\end{array}$

2.1.2. Curvas $\phi_{1}$ Constante no Plano $\mathrm{S}_{1} \mathrm{~V}_{1} \quad 30$

2.1.3. Ações para Aumento da Máxima Capacidade de Transmissão e de Controle de Tensão 31

2.1.4. Problema de Estabilidade de Tensão Devido ao Suporte Excessivo de Potência Reativa 34

2.1.5. Impedância Equivalente da Carga no Ponto de Máximo Carregamento 36

2.1.6. Existência de uma Potência "Maximum Maximorum" 38

2.1.7. Resumo 39

2.2. Avaliação e Reforço das Condições de Estabilidade de Tensão 40

3. Avaliação do Modelo Usual de Transformador com Tap Variável em Estabilidade de Tensão

3.1. Modelo Usual de Transformador com Tap Variável 42

3.1.1. Transformadores em Regime Permanente 42

3.1.2. Transformador com Tap Variável 43

3.1.3. Representação de Transformadores em Por Unidade 44

3.1.3.1. Exemplo: Vantagem da Representação em p.u. 44

3.1.3.2. Modelo Usual de Transformador com Tap Variável:

Transformador em Série com Impedância 45

3.1.3.3. Exemplo: Reintrodução do Transformador Ideal 47

3.1.3.4. Circuito П Equivalente ao Modelo Usual 51

3.1.3.5 Referências Iniciais sobre o Modelo Usual 53

3.2. Modelo Usual de Transformador com Tap Variável em Estudos

de Estabilidade de Tensão

4. Modelo Proposto para Transformador com Tap Variável e Impactos em Estudos de Estabilidade de Tensão $\quad 57$

4.1. Modelo Proposto $\quad 57$

$\begin{array}{ll}\text { 4.2. Exemplo } & 60 \\ \text { 4.3. Circuito } \pi \text { Equivalente ao Modelo Proposto } & 62\end{array}$

4.3. Circuito $\pi$ Equivalente ao Modelo Proposto 62

4.4. Modelo Proposto em Estudos de Estabilidade de Tensão 64

4.5. Comparação entre os Modelos Usual e Proposto em Estudos de
Estabilidade de Tensão 
5 Controle de Tensão através de Transformador com Tap Variável no Problema de Fluxo de Potência

5.1. Introdução

5.2. Modelagem Matemática $\quad 73$

5.2.1. Controle de Tensão no Modelo Usual 74

5.2.2. Controle de Tensão no Modelo Proposto 76

5.3. Limites dos Taps $\quad 80$

$\begin{array}{lr}\text { 6. Resultados } & \mathbf{8 1}\end{array}$

6.1. Teste em Laboratório $\quad 81$

6.1.1. Fluxo de Potência do Lado de Baixa para o Lado de Alta Tensão 81

6.1.2. Fluxo de Potência do Lado de Alta para o Lado de Baixa Tensão 82

6.2. Simulações Computacionais $\quad 85$

6.2.1. Sistemas Radiais $\quad 85$

$\begin{array}{ll}\text { 6.2.1.1. Sistema de 2 Barras } & 85\end{array}$

$\begin{array}{lr}\text { 6.2.1.2. Sistema de 4 Barras } & 90\end{array}$

$\begin{array}{lr}\text { 6.2.2. Sistemas Malhados } & 95\end{array}$

\begin{tabular}{lr} 
6.2.2.1. IEEE 14 Barras & 95 \\
\hline
\end{tabular}

6.2.2.2. IEEE 118 Barras 97

6.2.2.3. IEEE 30, 57 e 300 Barras 100

6.2.3. Controle de Tensão através de Transformador com Tap Variável 102

6.2.3.1. IEEE 14 Barras 103

6.2.3.2. IEEE 30 Barras $\quad 104$

6.2.3.3. IEEE 57 Barras 109

6.2.3.4. IEEE 118 Barras 111

$\begin{array}{ll}\text { 6.2.3.5. IEEE } 300 \text { Barras } & 113\end{array}$

$\begin{array}{ll}\text { 6.2.4. Sistemas Brasileiros } & 114\end{array}$

6.2.4.1. Sistema-Teste Brasileiro de 16 Barras $\quad 114$

6.2.4.2. Sistema-Teste Brasileiro de 33 Barras 121

6.2.4.3. Controle de Tensão por Meio de Transformador com Tap
Variável

6.2.4.3.1. Representação do Modelo Proposto 131

6.2.4.3.2. Controle de Tensão no Sistema-Teste Brasileiro de 16 Barras 132

6.2.4.3.3 Controle de Tensão no Sistema-Teste Brasileiro de 33 Barras 138

6.2.4.4 Índices de Estabilidade de Tensão 147

$\begin{array}{ll}\text { 6.2.4.4.1 Sistema-Teste Brasileiro de 16 Barras } & 147\end{array}$

6.2.4.4.2 Sistema-Teste Brasileiro de 33 Barras 149

6.3. Conclusões, Recomendação e Trabalhos Futuros 151

7. Conclusões e Próximos Estudos 152

$\begin{array}{lr}\text { Referências bibliográficas } & 155\end{array}$

Apêndice I - Circuitos П Equivalente de Transformador com Tap Variável

I.1. Circuito П Equivalente ao Modelo Usual de Transformador com

Tap Variável

I.2. Circuito П Equivalente ao Modelo Proposto de Transformador com Tap Variável 
Apêndice II - Exemplos da Literatura sobre Variação de Tap de Transformador com Tap Variável

II.1. Exemplos de Artigos

II.2. Exemplo de Relatório IEEE

Apêndice III - Impedâncias do Modelo Físico de Transformador com Tap Variável

III.1. Obtenção das Impedâncias através do Teste de Curto-Circuito no Transformador

III.2. Tentativa de Obtenção das Impedâncias através de Dois Testes de Curto-Circuito no Transformador

Apêndice IV - Impedâncias do Modelo Físico de Autotransformador com Tap Variável

IV.1. Conceitos Básicos sobre Autotransformador $\quad 175$

IV.1.1. Autotransformador Ideal 175

$\begin{array}{lr}178 & 178\end{array}$

IV.2. Determinacão das Impedâncias do Circuito Equivalente 181

Apêndice V - Tensões Críticas para Sistema de 2 Barras 181

V.1. Modelo Usual 181

V.2. Modelo Proposto 182

Apêndice VI - Comparação entre os Modelos Usual e Proposto de Transformador com Tap Variável nos Programas ORGANON e PSAT

VI.1. Comparação entre os Modelos através do Programa ORGANON 185

VI.2. Comparação entre os Modelos através do Programa PSAT 188 


\section{Lista de figuras}

Figura 2.1. Sistema de 2 Barras sem Qualquer Limitação 25

Figura 2.2. Curvas $P_{l}$ Constante no plano $\theta_{l} V_{l} \quad 26$

Figura 2.3. Curvas $Q_{l}$ Constante no plano $\theta_{l} V_{l} \quad 27$

Figura 2.4. Curvas $\phi_{l}$ Constante no plano $\theta_{l} V_{l} \quad 28$

Figura 2.5. Curvas $\mathrm{P}_{1}, \mathrm{Q}_{1}$ e $\phi_{1}$ Constantes no Plano $\theta_{1} V_{1}$

Figura 2.6. Curvas $P_{1 \max }, Q_{1 \max }$ e $\phi_{1}$ Constantes no Plano $\theta_{1} V_{1}$

$\begin{array}{lllllll}\text { Figura 2.7. Curvas } P_{l}, Q_{l} \text { e } \phi_{1} \text { Constante no Plano } \theta_{l} & V_{l} & \text { com } & \\ \text { Carregamento Maior que o Máximo } & & & & & 30\end{array}$

Figura 2.8. Curvas $\phi_{1}$ Constante no Plano $S_{1} V_{1} \quad 31$

Figura 2.9. Sistema de 2 Barras com Capacitor $\quad 32$

Figura 2.10. Curvas para as Situações Com e Sem Capacitor e Análise da Ação de Controle de Tensão com Ponto de Operação na Região Normal

Figura 2.11. Curvas para as Situações Com e Sem Capacitor e Análise da Ação de Controle de Tensão com Ponto de Operação na Região Anormal

Figura 2.12. Excesso de Compensação de Potência Reativa Levando o Ponto de Máximo Carregamento e a Região Anormal para a Faixa Usual de Operação das Tensões

Figura 2.13. Sistema de 2 Barras com Carga Modelada como Impedância Constante

Figura 2.14. Sistema Genérico de 2 barras

Figura 2.15. Equivalente de Thevenin do Sistema Genérico

Figura 2.16. Sistema de 2 Barras com Capacitor com Carga Modelada como Impedância Constante

Figura 2.17. Indicação das Impedâncias no Ponto de Máximo Carregamento nas Situações Sem e Com Capacitor

Figura 2.18. Existência de uma Potência Máxima mesmo com

Capacidade Infinita de Suporte de Potência Reativa

Figura 3.1. Modelo de um Transformador Operando em Regime Permanente

Figura 3.2. Modelo Simplificado de Transformador Operando em Regime Permanente

Figura 3.3. Representação de Transformadores em p.u. quando Valores-Base de Tensão obedecem a Relação de Transformação

Figura 3.4. Modelo Usual de Transformador com Tap Variável

Figura 3.5. Sistema de 3 Barras

Figura 3.6. Sistema de 3 Barras em p.u. 48

Figura 3.7. Sistema de 3 Barras em p.u. Modificado devido a Alteração do Tap

Figura 3.8. Sistema de 3 Barras em p.u. com Inserção do Transformador Ideal 
Figura 3.9. Circuito $\pi$ (com Impedâncias) Equivalente ao Modelo Usual de Transformador com Tap Variável

Figura 3.10. Circuito $\pi$ (com Admitâncias) Equivalente ao Modelo Usual de Transformador com Tap Variável

Figura 3.11. Curvas $\phi$ e Z Constante para Dois Valores de Tap com o Modelo Usual e com Fluxo da Barra $k$ para a Barra $m$

Figura 3.12. Curvas $\phi$ e Z Constantes para dois Valores de Tap com o Modelo Usual e com Fluxo da Barra $m$ para a Barra $k$

Figura 4.1. Modelo Físico de Transformador com Tap Variável

Figura 4.2. Modelo Físico de Transformador com Tap Variável em p.u com $\dot{Z}_{k}($ p.u. $)=\dot{Z}_{m}($ p.u. $)$

Figura 4.3. Modelo Proposto para Transformador com Tap Variável

Figura 4.4. Modelo Proposto para Transformador com Tap Variável com Reflexão de Impedância

Figura 4.5. Variação da Impedância com o Tap do Transformador para os Modelos Usual e Proposto

Figura 4.6. Sistema de 3 Barras com Modelo Físico do Transformador

Figura 4.7. Sistema de 3 Barras em p.u. com Modelo Proposto do Transformador com Tap Variável

Figura 4.8. Circuito $\pi$ (com Impedâncias) Equivalente ao Modelo Proposto

Figura 4.9. Circuito $\pi$ (com Admitâncias) Equivalente ao Modelo Proposto

Figura 4.10. Curvas $\phi$ e Z Constante para Dois Valores de Tap com o Modelo Proposto e com Fluxo da Barra $k$ para a Barra $m$

Figura 4.11. Curvas $\phi$ e Z Constantes para dois Valores de Tap com o Modelo Proposto e com Fluxo da Barra $m$ para a Barra $k$

Figura 4.12. Comparação entre os Modelos Usual e Proposto com Fluxo da Barra $k$ para barra $m$

Figura 4.13. Comparação entre os Modelos Usual e Proposto com Fluxo da Barra $k$ para barra $m$

Figura 5.1. Controle de Tensão Através de Transformador com Tap Variável

Figura 5.2. Modelo Usual com Admitância

Figura 5.3. Modelo Proposto Representado Através de Admitâncias 76

Figura 5.4. Modelo Proposto com Reflexão de Admitância 77

Figura 6.1. Curvas $\phi$ Constante para Dois Valores de Tap Obtidas em Laboratório e com Fluxo de Potência do Lado de Baixa para o Lado de Alta Tensão

Figura 6.2. Curvas $\phi$ Constante para Dois Valores de Tap Obtidas em Laboratório e com Fluxo de Potência do Lado de Alta para o Lado de Baixa Tensão

Figura 6.4. Comparação entre os Modelos Usual e Proposto com Fluxo da Barra 1 para Barra 2

Figura 6.5. Comparação entre os Modelos Usual e Proposto com Fluxo da Barra 2 para Barra 1 
Figura 6.7. Comparação entre os Modelos Usual e Proposto com Fluxo da Barra 1 para Barra 4 / Curvas Referentes à Barra 4

Figura 6.8. Comparação entre os Modelos Usual e Proposto com Fluxo da Barra 1 para Barra 4 / Curvas Referentes à Barra 3

Figura 6.9. Comparação entre os Modelos Usual e Proposto com Fluxo da Barra 1 para Barra 4 / Curvas Referentes à Barra 2

Figura 6.10. Comparação entre os Modelos Usual e Proposto com Fluxo da Barra 4 para Barra 1 / Curvas Referentes à Barra 1

Figura 6.11. Comparação entre os Modelos Usual e Proposto com Fluxo da Barra 4 para Barra 1 / Curvas Referentes à Barra 2

Figura 6.12. Comparação entre os Modelos Usual e Proposto com Fluxo da Barra 4 para Barra 1 / Curvas Referentes à Barra 3

Figura 6.13. Comparação entre os Modelos Usual e Proposto /Barra 5 / IEEE14 Barras

Figura 6.14. Comparação entre os Modelos Usual e Proposto / Barra 6 / IEEE14 Barras

Figura 6.15. Comparação entre os Modelos Usual e Proposto / Barra 17 / IEEE 118 Barras

Figura 6.16. Comparação entre os Modelos Usual e Proposto / Barra 30 / IEEE 118 Barras

Figura 6.17. Comparação entre os Modelos Usual e Proposto / Barra 37 / IEEE 118 Barras

Figura 6.18. Comparação entre os Modelos Usual e Proposto / Barra 38 / IEEE 118 Barras

Figura 6.19. Comparação entre os Modelos Usual e Proposto / Barra 30 / IEEE 30 Barras

Figura 6.20. Comparação entre os Modelos Usual e Proposto / Barra 31 / IEEE 57 Barras

Figura 6.21. Comparação entre os Modelos Usual e Proposto / Barra 9033 / IEEE 300 Barras

Figura 6.22. Comparação entre os Modelos Usual e Proposto / Barra 9 /

Controle de Tensão / IEEE 14 Barras

Figura 6.23. Comparação entre os Modelos Usual e Proposto / Transformador entre as Barras 4 e 9 / Controle de Tensão / IEEE 14 Barras

Figura 6.24. Comparação entre os Modelos Usual e Proposto / Barra 9 / Controle de Tensão / IEEE 30 Barras

Figura 6.25. Comparação entre os Modelos Usual e Proposto / Transformador entre as Barras 6 e 9 / Controle de Tensão / IEEE 30 Barras

Figura 6.26. Comparação entre os Modelos Usual e Proposto / Barra 12 / Controle de Tensão / IEEE 30 Barras

Figura 6.27. Comparação entre os Modelos Usual e Proposto / Transformador entre as Barras 4 e 12 / Controle de Tensão / IEEE 30 Barras

Figura 6.28. Comparação entre os Modelos Usual e Proposto / Barra 24 / Controle de Tensão / IEEE 30 Barras

Figura 6.29. Comparação entre os Modelos Usual e Proposto / Barra 27 / Controle de Tensão / IEEE 30 Barras 
Figura 6.30. Comparação entre os Modelos Usual e Proposto / Transformador entre as Barras 28 e 27 / Controle de Tensão / IEEE 30 Barras

Figura 6.31. Comparação entre os Modelos Usual e Proposto / Barra 32 / Controle de Tensão / IEEE 57 Barras

Figura 6.32. Comparação entre os Modelos Usual e Proposto / Transformador entre as Barras 32 e 34 / Controle de Tensão / IEEE 57 Barras.

Figura 6.33. Comparação entre os Modelos Usual e Proposto / Barra 50 / Controle de Tensão / IEEE 57 Barras

Figura 6.34. Comparação entre os Modelos Usual e Proposto / Transformador entre as Barras 10 e 51 / Controle de Tensão / IEEE 57 Barras

Figura 6.35. Comparação entre os Modelos Usual e Proposto / Barra 38 / Controle de Tensão / IEEE 118 Barras

Figura 6.36. Comparação entre os Modelos Usual e Proposto / Transformador entre as Barras 37 e 38 / Controle de Tensão / IEEE 118 Barras

Figura 6.37. Comparação entre os Modelos Usual e Proposto / Barra 9003 / Controle de Tensão / IEEE 300 Barras

Figura 6.38. Comparação entre os Modelos Usual e Proposto / Transformador entre as Barras 9001 e 9006 / Controle de Tensão / IEEE 300 Barras

Figura 6.39. Sistema-Teste de 16 Barras

Figura 6.40. Comparação entre os Modelos Usual e Proposto / Barra 4 / Sistema-Teste Brasileiro de 16 Barras

Figura 6.41. Número de Iterações para Obtenção de cada Ponto do Fluxo de Potência Continuado / Modelo Usual / Sistema-Teste Brasileiro de 16 Barras

Figura 6.42. Número de Iterações para Obtenção de cada Ponto do Fluxo de Potência Continuado / Modelo Proposto / Sistema-Teste Brasileiro de 16 Barras

Figura 6.43. Sistema-Teste de 33 Barras

Figura 6.44. Comparação entre os Modelos Usual e Proposto / Barra 960 / Sistema Teste Brasileiro de 33 Barras

Figura 6.45. Número de Iterações para Obtenção de cada Ponto do Fluxo de Potência Continuado / Modelo Usual / Sistema-Teste Brasileiro de 33 Barras

Figura 6.46. Número de Iterações para Obtenção de cada Ponto do Fluxo de Potência Continuado / Modelo Proposto / Sistema-Teste Brasileiro de 33 Barras

Figura 6.47. Sistema-Teste Brasileiro de 16 Barras com Barras Fictícias

Figura I.1. Circuito $\prod$ Genérico

Figura II.1. Efeito da Variação de Tap de Transformador com Tap Variável [36]

Figura II.2. Efeito da Variação de Tap de de Transformador com Tap Variável [37]

Figura II.3. Circuito do Exemplo

Figura II.4. Efeitos da Variação do Tap [38]

Figura II.5. Efeitos da Variação do Tap a Partir de (II.1) 
Figura III.1. Curto-Circuito para Determinação do Valor Total da Impedância apresentado em [24]

Figura III.2. Triangulo Referente ao Curto-Circuito Apresentado em [24]

Figura IV.1. Autotransformador Ideal

Figura IV.2. (A) - Autotransformador

(B) - Transformador

Equivalente

Figura IV.3. Circuito Equivalente de um Autotransformador

Figura IV.4. Circuito Equivalente de um Autotransformador Obtido através do Teste de Curto-Circuito

Figura IV.5. Circuito Equivalente de um Transformador Convencional (Sem Ligação Entre as Bobinas) obtido através do Teste de Curto-Circuito

Figuras IV.6. Transformador Convencional Equivalente ao Autotransformador

Figura VI.1. Sistema de 9 Barras apresentado em [50] com Barras Fictícias

Figura VI.2. Sistema de 14 Barras Apresentado em [51] com Barras Fictícias 


\section{Lista de tabelas}

Tabela 4.1. Comparação entre os Modelos Usual e Proposto Relativa à Margem de Estabilidade de Tensão

Tabela 4.2. Comparação entre os Modelos Usual e Proposto Relativa às Ações de Controle de Tensão

Tabela 6.1. Grandezas Referentes ao Caso-Base (A) e Comparação entre os Modelos Usual e Proposto ao Alterar o Tap para 1.1 p.u., com Fluxo de Potência da Barra 1 para a Barra 2

Tabela 6.2. Grandezas Referentes ao Caso-Base (B) e Comparação entre os Modelos Usual e Proposto ao Alterar o Tap para 1.1 p.u., com Fluxo de Potência da Barra 1 para a Barra 2

Tabela 6.3. Grandezas Referentes ao Caso-Base (A) e Comparação entre os Modelos Usual e Proposto ao Alterar o Tap para 0.9 p.u., com Fluxo de Potência da Barra 2 para a Barra 1

Tabela 6.4. Grandezas Referentes ao Caso-Base (B) e Comparação entre os Modelos Usual e Proposto ao Alterar o Tap para 0.9 p.u., com Fluxo de Potência da Barra 2 para a Barra 1

Tabela 6.5. Relatório com Solução do Fluxo de Potência / Dados de Barra / Sistema-Teste Brasileiro de 16 Barras / Modelo Usual

Tabela 6.6. Relatório com Solução do Fluxo de Potência / Dados de Linha / Sistema-Teste Brasileiro de 16 Barras / Modelo Usual

Tabela 6.7. Relatório com Solução do Fluxo de Potência / Dados de Barra / Sistema-Teste Brasileiro de 16 Barras / Modelo Proposto

Tabela 6.8. Relatório com Solução do Fluxo de Potência / Dados de Linha / Sistema-Teste Brasileiro de 16 Barras / Modelo Proposto

Tabela 6.9. Relatório com Solução do Fluxo de Potência / Dados de Barra / Sistema-Teste Brasileiro de 33 Barras / Modelo Usual

Tabela 6.10. Relatório com Solução do Fluxo de Potência / Dados de Linha / Sistema-Teste Brasileiro de 33 Barras / Modelo Usual

Tabela 6.11. Relatório com Solução do Fluxo de Potência / Dados de Barra / Sistema-Teste Brasileiro de 33 Barras / Modelo Proposto

Tabela 6.12. Relatório com Solução do Fluxo de Potência / Dados de Linha / Sistema-Teste Brasileiro de 33 Barras / Modelo Proposto

Tabela 6.13. Relatório do ANAREDE para Solução do Fluxo de Potência / Sistema-Teste Brasileiro de 16 Barras / Modelo Usual

Tabela 6.14. Relatório do ANAREDE para Solução do Fluxo de Potência / Sistema-Teste Brasileiro de 16 Barras / Modelo Proposto

Tabela 6.15. Informações sobre os Transformadores do Sistema-Teste Brasileiro de 33 Barras

Tabela 6.16. Relatório do ANAREDE para Solução do Fluxo de Potência / Sistema-Teste Brasileiro de 33 Barras / Modelo Usual

Tabela 6.17. Relatório do ANAREDE para Solução do Fluxo de Potência / Sistema-Teste Brasileiro de 33 Barras / Modelo Proposto Tabela 6.18. Relatório com Índices de Estabilidade de Tensão / Sistema-Teste Brasileiro de 16 Barras / Modelo Usual 
Tabela 6.19. Relatório com Índices de Estabilidade de Tensão / Sistema-Teste Brasileiro de 16 Barras / Modelo Proposto

Tabela 6.20. Relatório com Índices de Estabilidade de Tensão / Sistema-Teste Brasileiro de 33 Barras / Modelo Usual

Tabela 6.21. Relatório com Índices de Estabilidade de Tensão /

Sistema-Teste Brasileiro de 33 Barras / Modelo Proposto

Tabela VI.1. Tensões nas Barras do Sistema de 9 Barras / Relatório Fornecido pelo Programa ORGANON / Modelo Usual

Tabela VI.2. Carregamento dos Ramos da Rede do Sistema de 9 Barras / Relatório Fornecido pelo Programa ORGANON / Modelo Usual

Tabela VI.3. Resultados da Áreas do Sistema / Relatório Fornecido pelo Programa ORGANON / Modelo Usual

Tabela VI.4. Tensões do Relatório de Fluxo de Potência [43] para Sistema de 9 Barras

Tabela VI.5. Tensões nas Barras do Sistema de 9 Barras / Relatório Fornecido pelo Programa ORGANON / Modelo Proposto

Tabela VI.6. Carregamento dos Ramos da Rede do Sistema de 9 Barras / Relatório Fornecido pelo Programa ORGANON / Modelo Proposto

Tabela VI.7. Resultados da Áreas do Sistema de 9 Barras / Relatório Fornecido pelo Programa ORGANON / Modelo Proposto

Tabela VI.8. Relatório com Solução do Fluxo de Potência Fornecido pelo PSAT / Dados de Barra / Sistema de 14 Barras / Modelo Usual

Tabela VI.9. Relatório com Solução do Fluxo de Potência Fornecido pelo PSAT / Dados de Linha / Sistema de 14 Barras / Modelo Usual

Tabela VI.10. Tensões do Relatório de Fluxo de Potência [43] para Sistema de 14 Barras

Tabela VI.11. Relatório com Solução do Fluxo de Potência Fornecido pelo PSAT / Dados de Barra / Sistema de 14 Barras / Modelo Proposto Tabela VI.12. Relatório com Solução do Fluxo de Potência Fornecido pelo PSAT / Dados de Linha / Sistema de 14 Barras / Modelo Proposto 


\section{Simbologia e Siglas}

$V_{k} \quad$ Módulo da tensão na barra $k$;

$\theta_{k} \quad$ Ângulo da tensão na barra $k$;

$P_{k} \quad$ Potência ativa na barra $k$

$Q_{k} \quad$ Potência reativa na barra $k$

$S_{k} \quad$ Potência aparente na barra $k$

$Z_{t} \quad$ Módulo da impedância da linha de transmissão

$\alpha_{t} \quad$ Ângulo da impedância da linha de transmissão

$\phi_{1} \quad$ Ângulo do fator de potência

$X_{c} \quad$ Reatância capacitiva

$\dot{Z}_{\text {th }} \quad$ Impedância de Thevenin

$\dot{Z}_{s h} \quad$ Impedância shunt

$R_{k}+j X_{k} \quad$ Impedância série do lado $k$ do transformador, composta pela resistência do enrolamento e pela reatância de dispersão do referido lado

$R_{k}^{\prime}+j X_{k}^{\prime} \quad$ Impedância série do lado $k$ do transformador referida para o outro lado

$\mathrm{R}_{\mathrm{f}} \quad$ Resistência shunt do transformador referente às perdas no ferro

$\mathrm{X}_{\text {mag }} \quad$ Reatância de magnetização

$\mathrm{N}_{\mathrm{k}} \quad$ Número de espiras do lado $k$ do transformador

a Tap do transformador em p.u.

$\Delta P_{k} \quad$ Resíduo de potência ativa líquida na barra $k$;

$\Delta Q_{\mathrm{k}} \quad$ Resíduo de potência reativa líquida na barra $k$;

$I_{k m} \quad$ Corrente elétrica no ramo $\mathrm{km}$ no sentido de $k$ para $m$;

$I_{m k} \quad$ Corrente elétrica no ramo $\mathrm{km}$ no sentido de $m$ para $k$;

$P_{m k} \quad$ Fluxo de potência ativa da barra $m$ para a barra $k$;

$Q_{k m} \quad$ Fluxo de potência reativa da barra $k$ para a barra $m$;

$\gamma \quad$ Carregamento adicional utilizado no fluxo de potência continuado.

$P_{D_{k}} \quad$ Potência ativa demandada de uma barra $k$ qualquer

$Q_{D_{k}} \quad$ Potência reativa demandada de uma barra $k$ qualquer 
$P_{D_{k}}^{0} \quad$ Potência ativa demandada de uma barra $k$ qualquer no caso-base

$Q_{D_{k}}^{0} \quad$ Potência reativa demandada de uma barra $k$ qualquer no caso-base

LTC Load Tap Changer

OLTC On-Load Tap Changing

ULTC Under-Load Tap Changing

NLTC No-Load Tap Changing 